\title{
Histeropexia con Cinta de Dacrón
}

\author{
Universidad de Caldas - Facultad de Medicina \\ Hospital Universitario de Caldas \\ Departamento de Ginecología y Obstetricia \\ Dr. Mario Calle Mesa*
}

\section{INTRODUCCION}

Desde mayo de 1968 se empezó a utilizar en el Departamento de Ginecología y Obstetricia del Hospital Universitario de Caldas; la histeropexia con cinta de dacrón, descrita por Durfee (3) en 1966 para tratamiento del prolapso genital en mujeres jóvenes que desean conservar la función reproductora o que no aceptan la histerectomía por diversas razones.

El presente estudio se realizó con el propósito de evaluar nuestra experiencia con esta técnica sencilla y aportar alguna información adicional a la escasa literatura disponible sobre la materia.

\section{MATERIAL Y METODOS}

El material de estudio está constituido por 20 pacientes a quienes se les practicó histeropexia con cinta de dacrón, entre mayo de 1968 y julio de 1980.

Los criterios de selección de las pacientes fueron los siguientes:

1. Pacientes menores de 35 años.

2. Con prolapso genital de II y III grado.

* Jefe Depto. de Gineco.Obstetricia
3. Que deseaban conservar su función reproductora, o

4. Que deseaban conservar el útero por otras razones.

Para determinar el grado de prolapso se empleó la siguiente clasificación:

a) Histerocele $I$ :

el cuello desciende hasta la mitad de la vagina.

b) Histerocele II:

el cuello desciende hasta el introito vaginal, sin sobrepasarlo.

c) Histerocele III:

el cuello sobrepasa el introito vaginal.

Algunas de las pacientes presentaban además cistocele de I, II o III grado solo, o asociado a rectocele de grados variables.

El procedimiento quirúrgico consiste en lo siguiente:

1. Laparatomía de tipo Pfannestiel. (Fig. 1).

2. En la cara posterior del istmo uterino, se hace una pequeña ventana transversal, separando el peritoneo del miometrio. (Fig. 2). 
Figura No. 1

\section{IMCISION DE PFANNENSTIEL}



Figura No. 2

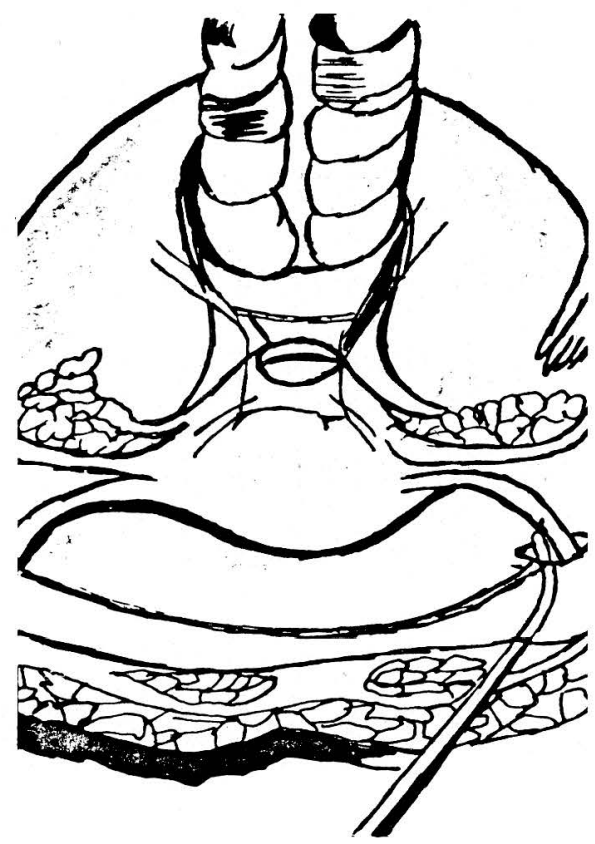

3. Se introduce una pinza de Pean, extra peritonealmente desde la salida abdo- minal del ligamento redondo, a través del canal inguinal. Luego, entre las hojas del ligamento ancho, se conduce el extremo de la misma hasta llegar a la apertura peritoneal a nivel del istmo uterino para tomar un extremo de la cinta de dacrón de $12 \mathrm{cms}$. de largo por $0,5 \mathrm{cms}$. de ancho y dejarlo reparado en el extremo de la herida quirúrgica, (Fig. 3 y 4 ).

Figura No. 3

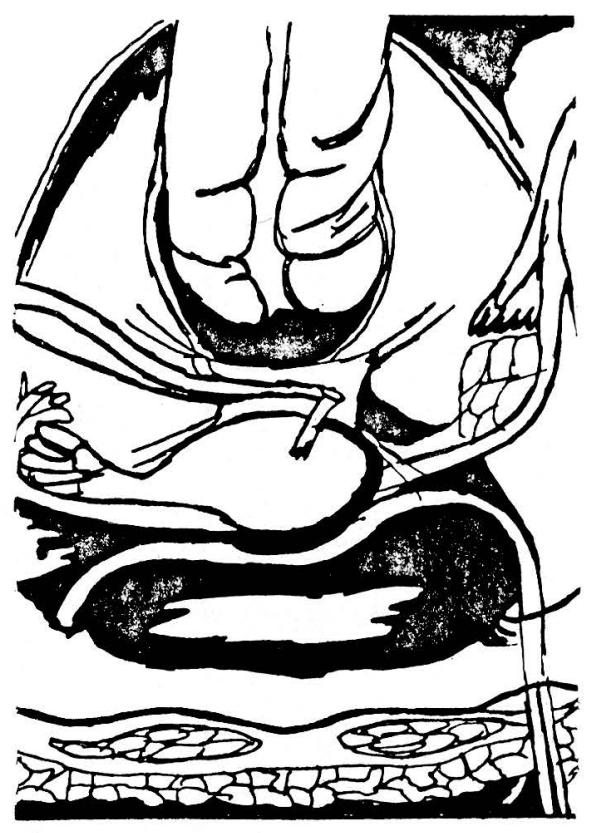

4. En el lado opuesto se repite el mismo procedimiento.

5. La cintilla se fija al útero con puntos separados de hilo de dacrón o de algodón y se recubre con el peritoneo previamente separado, por medio de una sutura con catgut simple. (Fig. 5 y 6). 
Figura No. 4

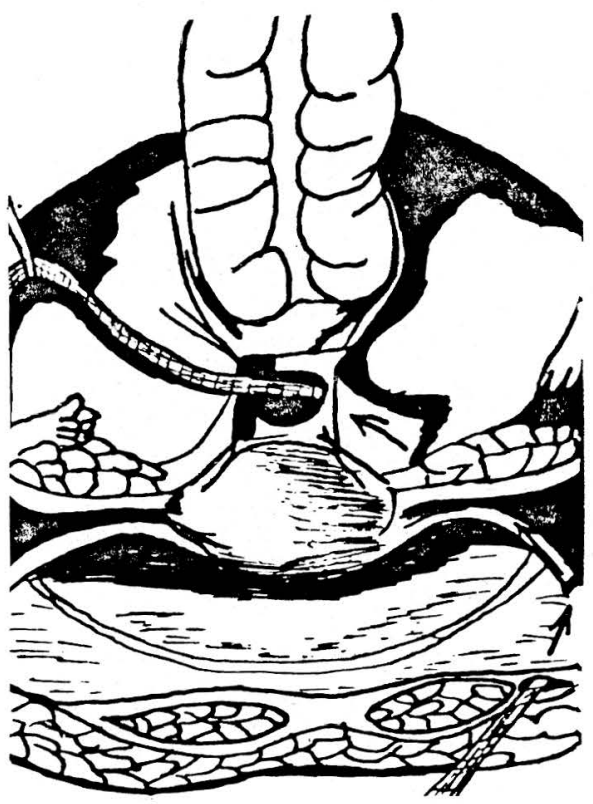

Figura No. 5

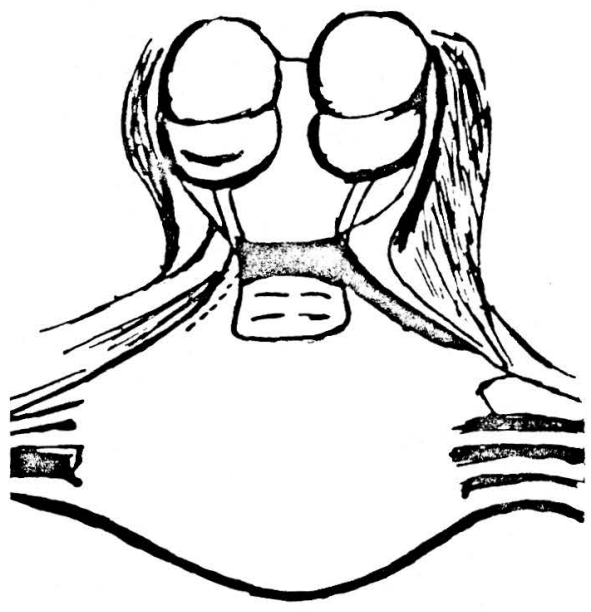

Figura No. 6

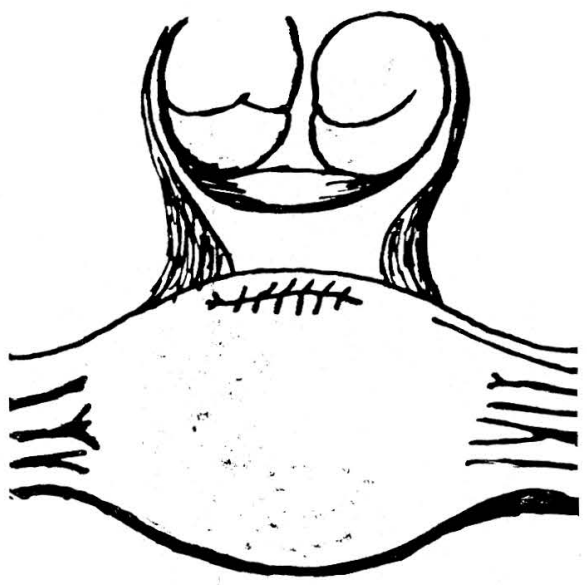

6. Los extremos de la cinta se fijan en ambos lados con puntos separados de hilo de dacrón o algodón a la cara interna de la aponeurosis del oblicuo menor y del recto del abdomen. (Fig. 7).

7. Debe ajustarse la tensión de la cinta, antes de suturarse en sus extremos, con el fin de no producir elevación excesiva del útero, o para que no quede muy floja, lo cual podría conducir a una corrección inadecuada del prolapso.

Se hizo un estudio de los siguientes aspectos: edad, paridad previa, tiempo de evolución del prolapso, procedimientos quirúrgicos empleados, evolución post-operatoria y controles ulteriores, resultado de la operación en cuanto al prolapso y en cuanto al curso y terminación de los embarazos post-operatorios.

Se analizan nuestros resultados en relación con los publicados en la literatura, se hacen algunos comentarios $y$ finalmente se definen conclusiones. 
Figura No. 7



\section{RESULTADOS}

Los siguientes son los resultados obtenidos en las 20 pacientes intervenidas en el Departamento de Ginecología y Obstetricia del Hospital Universitario de Caldas.

En 15 pacientes $(75 \%)$ la edad fue menor de 29 años. El promedio fue de

Tabla No. 1

DISTRIBUCION POR EDADES

\begin{tabular}{|ccc|}
\hline Grupos de edad & Número & Porcentaje \\
\hline $20-24$ & 6 & 30 \\
$25-29$ & 9 & 45 \\
$30-34$ & 5 & 25 \\
\hline TOTAL & 20 & 100 \\
\hline
\end{tabular}

PROMEDIO: 26.8 años
26.8 años, con un rango de 20 a 33 años. Estos resultados son similares a los encontrados por GARCIA M.J. y Cols. (5), pero más bajos que los hallados en los otros estudios publicados $(1,3)$.

Tabla No. 2

PARIDAD PREVIA

\begin{tabular}{|ccc|}
\hline Paridad & Número & Porcentaje \\
\hline 0 & 2 & 10 \\
$1-3$ & 12 & 60 \\
$4-6$ & 5 & 25 \\
7 & 1 & 5 \\
\hline TOTAL & 20 & 100 \\
\hline
\end{tabular}

PROMEDIO: 3

Doce de las pacientes $(60 \%)$ tenían entre uno y tres hijos. Dos eran nulíparas. Este porcentaje de nuliparidad es similar al encontrado en uno de los estudios (1).

Tabla No. 3

TIEMPO DE EVOLUCION DEL PROLAPSO

\begin{tabular}{|ccc|}
\hline Duración & Número & Porcentaje \\
\hline 1 año & 7 & 35 \\
$1-3$ años & 7 & 35 \\
$4-6$ años & 4 & 20 \\
7 años & 2 & 10 \\
\hline TOTAL & 20 & 100 \\
\hline
\end{tabular}

En 14 pacientes $\left(70^{\circ}{ }_{0}\right)$ la duración del prolapso osciló entre 1 y 3 años. En dos la evolución del mismo fue mayor de 7 años. No aparece información al respecto en los demás estudios publicados.

En 14 el prolapso fue clasificado como de II grado. 
Tabla No. 4

CLASIFICACION DEL PROLAPSO

\begin{tabular}{|ccc|}
\hline Prolapso genital & Número & Porcentaje \\
\hline Grado II & 6 & 30 \\
Grado III & 14 & 70 \\
& & \\
\hline TOTAL & 20 & 100 \\
\hline
\end{tabular}

S€ realizó histeropexia con cinta de dacrón, como único procedimiento quirúrgico, a 13 pacientes $(65 \%)$.

Tabla No. 5

TIPO DE INTERVENCION PRACTICADA

\begin{tabular}{|lcc|}
\hline Operación & Número & Porcentaje \\
\hline $\begin{array}{l}\text { Histeropexia solan nte } \\
\text { Histeropexia y colpopes } \\
\text { rineoplastia }\end{array}$ & 13 & 65 \\
$\begin{array}{l}\text { Histeropexia y pomeroy } \\
\text { Histeropexia y salpinguecs } \\
\text { tomía derecha }\end{array}$ & 3 & 15 \\
\hline TOTAL & 1 & 5 \\
\hline
\end{tabular}

A tres se les complementó la cirugía con esterilización por la técnica de Pomeroy, por solicitud de las mismas, debido a su alta paridad $(5,6$ y 8 hijos, respectivamente).

A otras tres, además de la histeropexia, se les practicó colpoperineoplastia.

Se efectuó control post-operatorio en 17 pacientes $(85 \%)$, catorce $(70 \%)$ fueron controladas por períodos que oscilaron entre uno y once años.
Tabla No. 6

DURACION DE! SEGUIMIENTO

\begin{tabular}{|ccc|}
\hline Controles & Número & Porcentaje \\
\hline 1 año & 3 & 15 \\
$1-3$ años & 9 & 45 \\
3 años & 5 & 25 \\
sin control & 3 & 15 \\
\hline TOTAL & 20 & 100 \\
\hline
\end{tabular}

Tabla No. 7

NUMERO Y TERMINACION

DE LOS EMBARAZOS EN 7 PACIENTES

\begin{tabular}{|ccl|}
\hline Pacientes & Embarazos & Terminación \\
\hline 1 & 1 & Parto normal \\
2 & 1 & Cesárea \\
3 & 1 & Parto con forceps \\
4 & 1 & Parto normal \\
5 & 1 & Cesárea \\
6 & 2 & Cesáreas \\
7 & 1 & Cesárea \\
\hline TOTAL & 8 & 8 \\
\hline
\end{tabular}

Siete de las pacientes $(41.2 \%)$ tuvieron ocho embarazos. Las que tuvieron parto normal, éste ocurrió en el hogar.

Se realizó cesárea en el $62.5 \%$ de las pacientes.

Tabla No. 8

RESULTADO DE LA INTERVENCION

\begin{tabular}{|lrc|}
\hline Resultado & Número & Porcentaje \\
\hline Satisfactorio & 18 & 90 \\
Recidiva del prolapso & 2 & 10 \\
\hline TOTAL & 20 & 100 \\
\hline
\end{tabular}


En el $90 \%$ (18 casos), el resultado de la intervención quirúrgica fue satisfactorio. Se reprodujo el prolapso en dos pacientes. A una se le practicó histerectomía vaginal un año después (tenía 32 años y 5 hijos). La otra no volvió a control.

Dos pacientes presentaron infección de la herida y eventración post-operatoria. Se les retiró la cinta de dacrón y se presentó recidiva del prolapso en una de ellas.

Una de las dos nulíparas, consultó por esterilidad 8 años después de la cirugía, comprobándose factor masculino como causa de aquella.

\section{COMENTARIOS}

La histeropexia con cinta de dacrón está indicada para la corrección del prolapso genital de II y III grado en mujeres jóvenes (menores de 35 años), que desean conservar la función reproductora o que no quieren que se les extirpe el útero.

Los estudios hasta ahora publicados $(1,3,5)$, coinciden con el presente, en estos aspectos.

Dos pacientes eran nulíparas, cifra similar a la encontrada por BAQUERO B. TUBERQUIA J.C. (1), lo cual confirma que en la etiología del prolapso genital, intervienen otros factores diferentes de la paridad.

En el estudio de GARCIA, M.J. y Col. (5) el $100 \%$ de las pacientes ten ían baja paridad (1 a 3 partos previos).

El prolapso de II grado $(30 \%)$ fue bastante menor que el encontrado en otros estudios, de $84 \%$ (1) y $92 \%$ (5).

La histeropexia como único procedimiento quirúrgico $(65 \%)$, realizada en este estudio es la más numerosa de las publicadas hasta el presente.

El $41.2 \%$ de las pacientes operadas, se embarazaron, tasa ésta más alta que la obtenida en otros estudios que varían entre $16.7 \%$ y $30 \%(5,3,1)$.

Se pracțicó cesárea en el $62.5 \%$ de las pacientes. En los otros estudios este porcentaje osciló entre el 50 y el $100 \%$ $(1,3,5)$.

Se hizo seguimiento en $17(85 \%)$; nueve $(45 \%)$ fueron controladas entre uno y tres años, con reproducción del prolapso por infección en una que no volvió a control.

Una paciente murió de cáncer de mama, a los seis meses de haber tenido un parto normal.

La que fue controlada durante 11 años no ha presentado recidiva del prolapso.

La histeropexia con cinta de dacrón, tiene sobre la operación de Manchester Fothergill, las ventajas de permitir realizar otros procedimientos quirúrgicos, en el mismo tiempo operatorio, como esterilización voluntaria, miomectomías, cirugía de trompas y de ovarios $(1,5)$, menor frecuencia de esterilidad, aborto y trabajo de parto prematuro (6), posibilidad de corregir la retroversión uterina y pronóstico más favorable sobre la fertilidad.

\section{CONCLUSIONES}

La histeropexia con cinta de dacrón, para la corrección del prolapso genital en mujeres jóvenes que desean conservar la función reproductora o no quieren que se les practique histerectomía, es la mejor técnica hasta ahora descrita, de acuerdo con los resultados obtenidos en el presente estudio y en los demás publicados, $(1,3,5)$. 
El procedimiento quirúrgico no interfiere con la fertilidad y aún cuando el parto por vía vaginal es posible, se recomienda la cesárea en todas las pacientes.

La técnica quirúrgica es sencilla, permite realizar simultáneamente otros procedimientos para tratar patología ginecológica y el dacrón es un material fuerte, bien tolerado y de fácil consecución en nuestro medio.

Después de la publicación de DURFEE en 1966, la casuística continúa siendo escaso en nuestro medio: 65 intervenciones en tres estudios.

Se recomienda su empleo a nivel nacional para comparar los resultados con otros estudios.
En nuestra opinión, las pacientes que presentan cistocele y rectocele de III grado, requieren el tiempo quirúrgico vaginal, pues la técnica no los corrige satisfactoriamente.

\section{RESUMEN}

Se presentan los resultados obtenidos en 20 pacientes jóvenes con prolapso genital, a quienes se les practicó corrección por medio de histeropexia con cinta de dacrón.

Se hace énfasis en la conservación de la fertilidad, la baja recidiva del prolapso, aún después del parto vaginal y la sencillez de la técnica. Se recomienda su empleo para este tipo de pacientes.

\section{HYSTEROPEXIA WITH DACRON RIBBON}

\section{SUMMARY}

This article presents the results in 20 young women with genital prolapse, who had correction through hysteropexia with Dacron ribbon.

\section{BIBLIOGRAFIA}

1. BAQUero B, TUBERQUIA J.C. Prolapso uterino en mujeres jóvenes tratadas con suspensión con dacrón. Rev. Col. Obst. Ginecol 30:547, 1972.

2. BERNAL B. Histeropexia en mujeres jóvenes. Ginecología y Obstetricia de México 33: 285-289, 1973.

3. DURFEE R.B. Operaciones de suspensión para tratar prolapso de órganos genitales. Clin. Obstet. Ginecol. México, Editorial Interamericana S.A., 1966, pp. 1047 - 1060.
Great emphasis is made on conservation of fertility and low relapse, even after vaginal delivery, and on the simplicity of this technique, highly recommended in these cases.

4. EMGE L.A., DURFEE R.B. Prolapso de órganos pélvicos. Cuatro milenios de tratamiento. Clin Obstet Ginecol. México, Editorial Interamericana S.A., 1966, pp. $997-1.031$.

5. GARCIA M.J.,URIBE E.G.,TOBON L.G. Relajación del peso pélvico. Suspensión con cinta de dacrón. Rev. Col. Obstet. Ginecol 31: 395, 1980.

6. GORDON B.G. Operación de Manchester. Clin Obstet Ginecol. México, Editorial Interamericana S.A., 1974, pp. 3 - 28. 